\title{
artículos
}

\section{Hacia un concepto de formación de usuarios y propuesta de un programa}

\author{
José Alfredo Verdugo Sánchez ${ }^{\star}$
}

\begin{abstract}
RESUMEN
El trabajo aborda los aspectos relacionados con el marco conceptual de la formación de usuarios de la información; en este sentido, se discuten los distintos puntos de vista que han vertido algunos autores, desde la óptica pedagógica. En ello se relaciona el concepto "formación" con el empleo que se ha hecho de él, en las distintas bibliotecas universitarias al formular acciones tendientes a formar usuarios.
\end{abstract}

Al relacionar el marco conceptual descrito con una posible práctica, se presenta de manera propositiva un programa universitario de formación de usuarios, dirigido al usuario de nivel licenciatura; concretamente a aquél matriculado en universidad de carácter público.

\section{ABSTRACT}

$\boldsymbol{O}_{n}$ the one hand, this paper deals with the conceptual framework of the formation of information users. Some authors's points of view of varied nature are discussed with a pedagogical perspective. The concept "formation" is related to its usage indifferent university libraries which devise activities aimed at his formation of users.

On the other hand, within the conceptual framework described above, a university program for the formation of users is proposed. This program is addressed to undergraduate students of public universities.

$P^{\prime}$ ara introducirnos en la significación real del problema, habrá que abordar y conceptuar desde arios enfoques, el término general "formación". En el análisis que de algunos autores realiza Honore ${ }^{1}$ sobre el particular, hemos encontrado que la formación designa "algo que se tiene, algo adquiri- do, algo de valor para el que dispone de ello". En este sentido algunos estudiosos llaman y determinan como formación a un recorrido que siempre han seguido, a un conjunto o serie de ejercicios que con antelación han realizado; más concretamente: a una experiencia adquirida.
Considerada desde el punto de vista del individuo, del sujeto, se entiende a la "formación" como una característica propia y particular de la persona, donde los antecedentes de ésta son los que van a determinar la forma, el alcance y el nivel de una formación dada. Sobre ello, Lhotellier ${ }^{2}$ se expresa acer-

* Subdirector de Servicios Bibliotecarios de la Universidad Pedagógica Nacional 
ca de la "formación", diciendo que "es la capacidad de transformar en experiencia significativa los acontecimientos cotidianos generalmente sufridos, en el horizonte de un proyecto personal y colectivo". En tal circunstancia, "no es algo que se posee, sino una aptitud o una función que se cultiva, y puede eventualmente desarrollarse".

De esta manera, la "formación" es como una actividad mediante la cual se buscan, a través de otro, las condiciones para que un saber recibido del exterior, posteriormente sea sintetizado y superado e igualmente, exteriorizado y superado e igualmente exteriorizado de nuevo, enriquecido hasta generar otra actividad; esto es, producir un ciclo cada vez con más y mejor retroalimentación.

Por "formación" también se ha entendido "la emergencia de un cierto tipo de persona que condensa fragmentos particulares de experiencia, es decir, la superación de la contradicción sujeto-objeto, en la medida en que la persona y solamente ella sintetiza estas dos entidades, utiliza activamente los momentos pasivos de su experiencia y se observa pasivamente su actividad y la de los demás, hasta el período en que la observación llega a ser ella misma acto, y así sucesivamente". ${ }^{3}$

Los estudios teóricos que muchos especialistas han realizado sobre el fenómeno de la formación denotan una fuerte vinculación con los procesos de enseñanza-aprendizaje; tanto, que se hace necesario retomar esta posible vinculación y sentar las bases para el deslinde de alcances y limitaciones entre una y otra acción.

De esta forma, por ejemplo, Menze4 nos invita a reflexionar cuando dice que la formación no es ni un concepto general que abarque la educación y la enseñanza, ni tampoco subordinado a estos. Se puede indicar algún sentido de este término mediante su distinción del de educación, ya que ambos se hallan en cierta correlación. La conjunción y similitud de ambos y términos es la que determina el quehacer de le pedagogía. Así, la ecuación se refiere al carácter y a la acción. La formación abarca la inteligencia de sí y del mundo que tiene el hombre.

Desde el punto de vista de la pedagogía actual, el concepto de "formación" también connota distintas acciones: por un lado, el proceso de desarrollo en el cual se manifiesta al mundo exterior parte del mundo interior del individuo. Por otro lado, la "formación" forzosamente se da en un nivel de intercambio con el mundo real que rodea a cada ser. No se manifiesta como un proceso orgánico que puede efectuarse por sí mismo. Requiere necesariamente de un proceso educativo previo o simultáneo.

La diversidad de enfoques cuando se habla de "formación", radica, según lo expresado por Honore, ${ }^{5}$ en que el término "formación", tal y como se emplea hoy día, se refiere principalmente a actividades cuyo objetivo pocas veces es preciso y el resultado de tal actividad no es evaluado.

Dada la experiencia repetitiva del empleo del término, todo aquél que ha recurrido a la "formación", por una u otra causa, considera de ella muy diversos aspectos. La problemática básica radica en que aun considerando estos aspectos diversos no se entiende desde que puntos de vista se da el enfoque: si es desde el punto de vista del objeto (exterioridad) o desde el punto de vista del sujeto (interioridad), o más aún, si es desde el punto de vista de ambos: de la relación sujeto-objeto.

Es importante considerar la reflexión de Menze, 6 cuando dice que "la formación multiplica las oportunidades de instrucción porque hace al hombre es más ágil y más perspicaz, porque amplía su horizonte y le descubre nuevas posibilidades... si por un lado la enseñanza ha venido a ser un medio imprescindible de formación, en cambio, la formación resultante de la enseñanza protege al hombre contra la estrechez de la especialización (...)

No puede darse nunca por terminada (la formación), no es un suplemento de la existencia humana, al que se pueda renunciar, sino que más bien fundamenta la posibilidad de una existencia humana en general".
Dentro del amplio espectro de los distintos estudiosos del tema que han abordado el concepto de "formación", están aquellos que apuntan a la formulación de una teoría de la formación, considerando para ello los distintos pensamientos pedagógicos de actualidad.

Entre las aproximaciones teóricas más importantes, podemos destacar: la Teoría de la formación formal; la Teoría de la formación categorial; la Teoría dialogista de la formación y la Teoría de la formación técnica.

Cada una de estas teoría propuestas desde muy iniciado el siglo XIX hasta la segunda parte del XX, dan suma importancia a aspectos distintos del hombre, aunque en su conjunto todas ellas encierran una verdadera preocupación por la superación del individuo,7 quien será siempre el motor en torno al cual la pedagogía fundamente sus propuestas teóricas; incluidas en ellas a la "formación".

Como queda implícito en la literatura, el amplio y complejo uso y manejo que se ha hecho de la "formación", aplicada en uno y otro sentido: como aspecto involucrado en el proceso enseñanza-aprendizaje, o bien como apartado independiente de este proceso, no ha propiciado el establecimiento de un criterio unificado en este sentido. Sin embargo es claro advertir que la formación aplicada en cualquier sentido a los usuarios de la información, implica un conocimiento previo, el cual de manera categórica estará otorgado por el nivel educativo del individuo; ya sea que se trate de un nivel concluido o bien de un nivel en curso.

En aplicación directa al ámbito de la biblioteca y demás unidades de información, la formación de usuarios de información debe entenderse como el conjunto de actividades pedagógicas que, expresadas en términos graduales dentro de un proceso de enseñanza aprendizaje, están encaminadas a la óptima explotación de los recursos de información necesarios y suficientes (por parte del usuario) para la resolución de algún requerimiento académico o de cualquier otra índole. 
Entendida de esta manera, la "formación" proporcionará al usuario los elementos y herramientas que le permitan (en un tiempo presente o futuro): determinar (en primera instancia) y localizar (después) cualquier recurso informativo de su interés.

Esto es, que el usuario de la información experimente por si mismo el hecho de involucrarse de manera directa en la consecución de su satisfactor de tipo informativo; pudiendo ser en un sistema organizado como la biblioteca el centro de documentación, el de información, el archivo, etcétera; o bien en cualquier acervo o colección que carezca de un proceso de sistematización bibliotecaria.

En nuestro país, el fenómeno de formación de usuarios de la información se ha manifestado de distintas maneras y formas. Ello ha reflejado distintas causas y efectos: en el primer caso podemos observar que muchos de los recursos de información que proporcionan nuestras universidades, por ejemplo a través de sus bibliotecas, centros de documentación, de información, archivos, librerías, etcétera, no son utilizados al máximo por los estudiantes matriculados.

En este tenor, muchas pueden ser también las razones de tal situación; una de ellas es, sin lugar a dudas, la escasa formación que el estudiante universitario recibe con respecto a la información, sus procesos, importancia, formas y modos de obtenerla.

Otra causa importante que refleja de manera sistemática la comunidad estudiantil universitaria, es el escaso hábito a la lectura que ha logrado desarrollar desde edad temprana, lo cual trae como consecuencia un limitado uso de la información que albergan las distintas bibliotecas universitarias del país. Lo anterior invariablemente se observa en aquellos estudiantes que solamente acuden a las bibliotecas (ya sean universitarias o públicas) a consultar materiales, que únicamente ayudan a resolver un trabajo escolar.

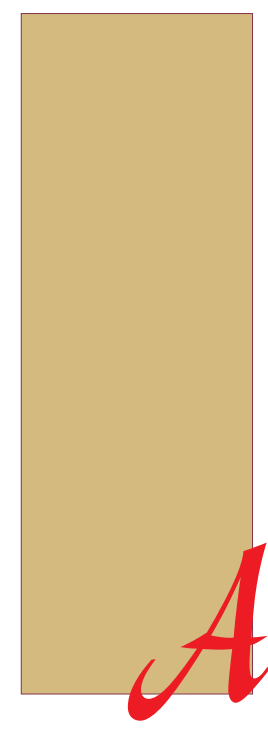

De alguna manera, la necesidad de cumplir y cubrir las exigencias de un plan de estudio obliga al estudiante universitario a involucrarse en la actividad de las propias bibliotecas y demás unidades de información, situación que de alguna manera ayuda a promover y cultivar el ejercicio hacia ese hábito no desarrollado de lectura.

Como efecto de estas causas, ya localizadas en las distintas bibliotecas universitarias, se ha determinado una serie de actividades encaminadas hacia la formación del usuario. En tales actividades encontramos una diversidad de enfoques: desde aquella denominada orientación, pasando por otras como son la instrucción, capacitación, formación, hasta la determinada como educación. Cualquiera que sea el término que la identifique, el grueso de sus objetivos y contenidos se enmarcan en lo que ahora podemos determinar como formación de usuarios.

En nuestro país, el fenómeno de formación de usuarios de la información se ha manifestado de distintas maneras y formas

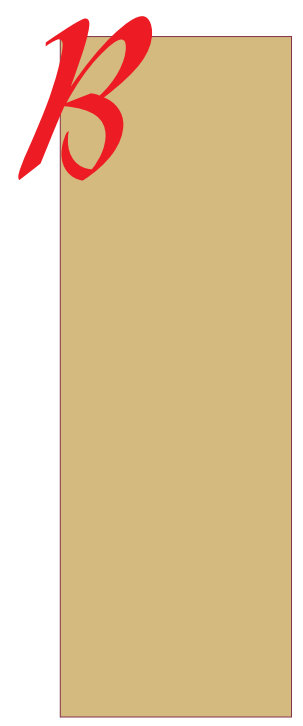

Pese a los esfuerzos realizados en este sentido por un gran número de instituciones, los resultados obtenidos no son los que pudieran esperarse, dado que la participación del usuario es sumamente limitada, tal como ha ocurrido en las Bibliotecas Departamentales de la UNAM $^{8}$

Un primer acercamiento de observación directa ${ }^{9}$ para la detección de tal situación, con estudiantes de distintos niveles en las diversas escuelas y facultades de la UNAM indicó que un alto porcentaje de estos, no sólo desconocen la biblioteca, sino que ignoran que existen otras fuentes de consulta además del tradicional libro de texto, y que la biblioteca o el centro de documentación, al archivo, la librería, etcétera, representan otra posibilidad más en la búsqueda y localización de la información.

Una muestra posterior ${ }^{10}$ recogida a través de la aplicación de un cuestionario para recabar información sobre las diversas actividades de formación que las bibliotecas tenían proyectadas, mostró que en aquellas universidades donde se habían realizados algunas actividades tendientes a la formación del estudiante en el uso y manejo de la in- 
formación, el comportamiento del usuario con respecto a la importancia de la biblioteca y sus recursos, era altamente positiva.

En términos generales, la "formación de usuarios" en México debe ser considerada como una actividad necesaria e importante para la vida académica del estudiante, por un lado, y por el otro, debe estimarse que con ella la biblioteca está promoviendo los servicios de información de manera amplia y masiva, así como el hábito a la lectura en la comunidad universitaria.

Paradójicamente, y pese a que la gran mayoría de las universidades del país contempla algún tipo de actividad para ayudar al usuario en la recuperación de la información, son escasas aquellas instituciones que han definido un programa formal con la concepción que en estas líneas se ha dado a la formación de usuarios.

Pese a su relevancia dentro de los sistemas de información, el usuario no ha encontrado en las bibliotecas universitarias la satisfacción plena de sus necesidades de información, Muchos argumentos se han esgrimido al respecto: destacan las limitaciones económicas, la carencia de personal especializado, las colecciones limitadas, entre otros.

No se puede negar que la influencia de estos señalamientos minimiza la satisfacción del usuario que desea y busca algún tipo de información. Pero también lo es el hecho de que el usuario ha tenido poca oportunidad de ser orientado correctamente en el uso y manejo de las fuentes y herramientas que los sistemas de información ponen a su disposición.

Resulta sumamente importante que el usuario de la información sepa y conozca todos aquellos elementos mínimos necesarios que le permitan encontrar por sí mismo lo que requiere, ya sea que se trate de un lugar específico (la biblioteca, el centro de información, de documentación, el archivo, un módulo, etcétera), o un tipo de material (un libro de texto, una enciclopedia, un diccionario, una revista, un diario, un índice, etcétera), o bien en el formato que sea ( un fonograma, un vi- deo, un material impreso, un cartel, un disco compacto, etcétera).

En ocasiones no es tan cierto que las bibliotecas universitarias no cuenten con los materiales o con las posibilidades de hacer llegar al usuario la información requerida; se conoce que en muchos casos, se debe a que la propia universidad no le ha enseñado al usuario las posibilidades de encontrar (dónde, como, cuándo y con quién, lo que él sabe que necesita.

Es la "formación del usuario" un elemento que aún no hemos podido rescatar e integrar al proceso formativo-educativo del estudiante universitario; elemento que abre un abanico de posibilidades reales para el usuario como demandante, y para el bibliotecario como facilitador, durante y después de los estudios profesionales.

La literatura que refleja la importancia de la formación del usuario en bibliotecas universitarias, establece claramente que no existen programas definidos tendientes a esta formación, por parte de los sistemas bibliotecarios. En la mayoría de los casos, los reportes que reflejan alguna actividad práctica se refieren a acciones aisladas que no están contempladas en un programa general con objetivos y metas determinadas., Tal parece que la "formación del usuario", así entendida, sólo se da en el momento que éste requiere algún servicio o tiene ante sí una demanda (que más bien se convierte en urgencia) de información.

La aplicación de la encuesta, 11 que de forma estratificada se realizó en diferentes universidades públicas de la República Mexicana, arrojó datos que por sí mismos son importantes. Del total de escuelas y facultades en que se practicó la encuesta tanto en el Distrito Federal como en algunos estados, se obtuvo que un alto porcentaje de bibliotecas incluían algunas "ideas" de "formación" al usuario. Así, por ejemplo, de las 29 bibliotecas departamentales encuestadas en la UNAM, un 70\% reportó incluir actividades en este sentido.
Sin embargo, es también significativo que cuando se trató de obtener copia o ejemplares de los programas que la biblioteca citaba, estos no existían. En este mismo sentido, y con el propósito de encontrar algún tipo de cruce en la información proporcionada por la biblioteca también se aplicó una encues$\operatorname{ta}^{12}$ a usuarios de la biblioteca en cuestión.

Las respuestas de los estudiantes encuestados (que a decir de la biblioteca ya se habían sometido al "programa de formación" de usuarios) distaron mucho de lo que se esperaba, resultando que:

Un alto porcentaje (el 69\%) de los 129 encuestados mostró grandes deficiencias en sus respuestas. Aun cuando ya habían cubierto el entrenamiento de biblioteca y pasado más de un semestre en contacto con los servicios de ésta, los estudiantes no conocían la mayor parte de los servicios que la biblioteca reportó. Tampoco mostraron habilidades en el uso y manejo de herramientas tan simples y cotidianas como lo son el catálogo al público, el cardes, o bien la localización de materiales en la estantería.

Si a todo esto le sumamos el desconocimiento de la obras de consulta básica de su especialidad y el nulo manejo de ellas, y más aún, el desinterés del propio usuario en conocerlas, estaremos hablando de la urgencia de establecer programas institucionalmente formales que abatan esta problemática.

También se ha observado que cuando la formación se deja a la buena voluntad del alumno (usuario), los resultados son más que desalentadores, empezando por la poca asistencia y participación del usuario, tal como ha ocurrido en años anteriores en la Biblioteca central de la propia UNAM y en la mayoría de las bibliotecas universitarias del país. ${ }^{13}$

La aplicación de la encuesta tuvo como característica principal que fue dirigida a alumnos de los primeros semestres de escuelas o facultades, tales como: Filosofía y Letras, Economía, Derecho, Ciencias Políticas, Arquitec- 
tura, Ciencias, Ingeniería, Química y Medicina.

De las mismas bibliotecas que fueron encuestadas en estas mismas condiciones en algunos de los estados de la República, encontramos que, por ejemplo, tanto la Universidad Autónoma de Baja California Sur (UABCS) como la Universidad de Colima (UC) ocuparon un porcentaje mayor al $90 \%$ de respuestas positivas con respecto al conocimiento que tienen de los servicios bibliotecarios en general.

En ambos casos, las acciones emprendidas por la biblioteca, si bien no responden a un programa de formación, tal como se ha expresado en líneas anteriores, por lo menos si quedó implícito que a los estudiantes a los cuales estaban dirigidas dichas acciones cumplieron su objetivo.

Las respuestas denotan menos desfase entre lo que la biblioteca dice hacer para sus usuarios y lo que sus usuarios dicen saber acerca de la biblioteca y sus servicios. Se encontró una comunión muy estrecha entre el binomio biblioteca-usuario.

Factor importante en estos casos lo constituye el hecho de constatar la existencia de programas de: visitas guiadas, carteles, periódico mural, pláticas, trípticos, etcétera, como lo demostraron la UABCS y la UC.

Otras universidades encuestadas en sus sistemas bibliotecarios, al igual que sus usuarios, como es el caso de la Universidades Autónomas de Nuevo León, de Sonora, Guerrero, Estado de México y Chiapas mostraron una gran deficiencia en este apartado. Las acciones son verdaderamente incipientes y la carencia de programas indica que la biblioteca está más preocupada por los procesos internos (técnicos y administrativos) que por la formación del usuario en el uso y manejo de los recursos informativos.

Como ya se ha apuntado, las razones de esta situación pueden ser de muy variadas causas. Lo sorprendente de la encuesta en estos casos es que la propia biblioteca desconoce la existencia y posibilidades de las acciones de un programa de formación de usuarios.

Casi en su totalidad, las bibliotecas universitarias de la República Mexicana que proporcionan alguna "formación" a sus usuarios, se concretan a la conocida y socorrida visita guiada y, en el mejor de los casos, se acompaña de algún paquete audiovisual o un diaporama; otras más complementan su formación con un tríptico, tal vez con alguna conferencia o con una plática informal, etcétera, con lo cual se limitan a informar aspectos generales del servicios bibliotecario que proporcionan sin modificar con ello la conducta o actitud de su usuario con respecto al uso, manejo e importancia de la información.

Dada la información recabada en el resultado de la encuesta, es importante señalar que las actividades programadas para la promoción de la formación del usuario universitario tienen una característica peculiar: la no normatividad de sus programas de formación.

Aun cuando en términos generales todas las universidades de carácter público observan los mismos objetivos, todos ellos dirigidos a la docencia, a la investigación y a la difusión de la cultura, los objetivos expresados en aquellas acciones detectadas, tendientes hacia una formación del usuario, son diametralmente distintos.

Una confusión muy recurrente en muchas de las actividades programadas es la formulación de cursos, folletos, trípticos y demás recursos en los cuales el contenido refleja una carga considerable de elementos técnicos de bibliotecología. El usuario de la información no requiere ni demanda ser bibliotecario, él requiere que se le indique la forma y métodos idóneos para llegar a su satisfactor informativo; bien sea una unidad de información, un libro, un módulo, un disco, un abstract, etcétera. Las precisiones de carácter técnico, que lejos de ayudar lo confunden, levantan en el usuario, una especie de barrera, que poco le permite interactuar con la biblioteca y con la información.

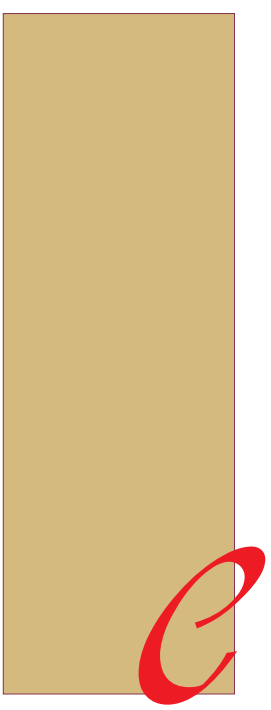

\section{El usuario de la información no requiere ni demanda ser bibliotecario, él requiere que se le indique la forma y métodos idóneos para llegar a su satisfactor informativo}

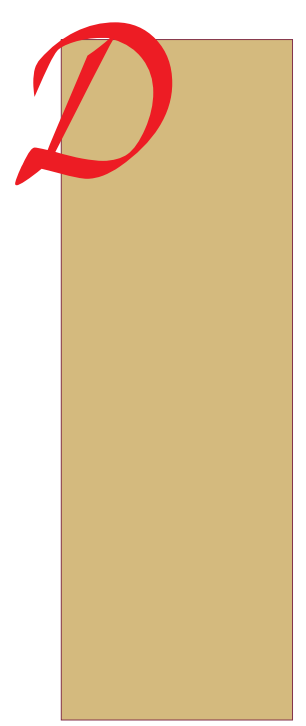


Como constante generalizada de la información obtenida en las bibliotecas universitarias se observó que la evaluación (parte importante de todo proceso de formación) no está presente.

Se ofrecen las acciones; se proporcionan las pláticas, se programan y conducen las visitas; se distribuyen los folletos, trípticos y carteles, se imparten los cursos, etcétera, pero la biblioteca no obtiene retroalimentación de su trabajo, por lo que la misma biblioteca no tiene elementos para discernir su actuación y mucho menos cuenta con parámetros que le permitan medir el alcance y limitaciones de sus programas. Una de las actividades que resulto singular es la recientemente reportada por la Biblioteca Central de la UNAM: el uso del video para dar a conocer de manera sintetizada, atractiva y clara, las posibilidades que el usuario tiene ante sí, con respecto a los servicios de información, deja abierta las opciones de transmisión y comunicación a grandes núcleos de usuarios. ${ }^{14}$

En este caso, estamos hablando de una técnica de efectos masivos y con impacto multiplicador, ya que además, el video está acompañado de un folleto explicativo que refuerza lo explicado gráficamente.

Todo lo anterior refleja que las distintas actividades que las bibliotecas formulen en pro de un mejor desarrollo y desempeño del usuario, ante los servicios de información, deben estar bien dirigidas, con objetivos claros y precisos; un contenido lógico y gradual, así como de una infraestructura mínima que permita responder satisfactoriamente a las necesidades y demandas del usuario.

En relación con los planteamientos de carácter teórico que se han vertido en líneas anteriores y a la constante encontrada en las distintas bibliotecas universitarias, en el sentido de formular alguna acción relacionada con la formación del usuario de la información, se ha considerado de manera propositiva, un programa que bien pudiera servir de lineamiento general aquellas bibliotecas universitarias que mantengan el interés de desarrollar en el usuario el buen uso y manejo de la información.

\section{PROPUESTA DE PROGRAMA}

La interacción de los individuos dentro de un grupo social, y el propio desarrollo de la sociedad, hacen posible la generación de gran cantidad de información, la cual al transcurrir el tiempo se convierte en conocimiento y pasa a formar parte de la cultura del propio grupo social. La información será, por un lado, instrumento que permite acceder, interpretar y modificar el conocimiento, y por el otro, será producto del mismo conocimiento. En este sentido, la importancia de la información radica en que a través de ella conoceremos a la sociedad en la que estamos inmersos, y estaremos en posibilidad de adaptarnos, o en otro caso, de transformarla o modificarla. Lo anterior se señala debido a que la información se encuentra presente en las actividades inherentes al desarrollo del ser social.

En la medida en que el individuo esté correctamente informado, tendrá más y mejor oportunidad de integrarse a su contexto social por tanto de conocerlo y participar en él, logrando su adaptación y desarrollo.

Por ello, es imprescindible que el ser humano esté en posibilidades de evolucionar a la par de la cultura, se le ofrezcan los medios y recursos para obtener la información; sistematizarla y utilizarla de manera eficaz.

El mundo de hoy, caracterizado por el constante cambio científico, tecnológico y social, así como por la rapidez con que ocurren estos cambios, ha hecho que los medios, formas y maneras de rescatar la información sean complejos. Es pues notoria la importancia que reviste la información en el desarrollo político, social, económico y cultural de un pueblo.

La crisis económica que afecta a las sociedades del mundo actual, y a nuestro país en particular, obliga a planear cuidadosamente las posibilidades y expectativas para el desarrollo; por ello, se producen los recortes presupuestales en áreas no prioritarias y priorita- rias (según lo entienden los planificadores de la política estatal). Estamos en presencia de la "economía de la austeridad". Dada esta situación, es insoslayable evitar el derroche, el desperdicio, el dispendio y la distracción de los escasos recursos presupuestarios y por ende aprovechar y usar de manera óptima y eficaz los recursos disponibles. Sobre todo, si estamos hablando del sector educativo y particularmente de nuestras universidades.

En este contexto se debe considerar a la información como un recurso valioso en sí mismo y que dependerá del uso que se haga de ella para que contribuya al desarrollo del país. "El valor de la información depende principalmente de los beneficios que deriven de su correcta utilización; por lo que es indispensable, para un país como el nuestro, adquirir el conocimiento o habilidades necesarias para localizar, evaluar, comunicar y usarla efectivamente". ${ }^{15}$

La información, fenómeno de expresión social, puede estar contenida en diferentes medios, tales como: la radio, la televisión, el libro, la revista, el periódico, el videograma, los fonogramas, el cine, el teatro, etcétera. Formas de preservar el conocimiento que pueden estar contenidas, organizadas y sistematizadas en la biblioteca u otros centros con características similares.

Para que el conocimiento contenido en los acervos pueda ser accesado, es decir que la información pueda ser rescatada es primordial que los estudiantes en todos los niveles, desarrollen las aptitudes y habilidades y cuenten con la formación necesaria que redunde en la óptima explotación de los recursos de información, suficiente para resolver algún requerimiento académico; esto es, que el estudiante pueda romper las posibles barreras que pudiera encontrar en la consecución de la información requerida.

A través de esta formación, el estudiante-usuario adquirirá la habilidad para utilizar al máximo los variados recursos de información; estará entrenado en el uso de las bibliotecas, centros de información, de documentación, etcétera, desarrollando actitudes hacia 
ellos y comprenderá la importancia de la búsqueda de la información y la instrucción bibliográfica.

En cualquier área del conocimiento, el uso y manejo de la información debe considerarse como contenido indispensable para el mayor desarrollo y mejor aprovechamiento del proceso enseñanza-aprendizaje. Por ello la formación del usuario en el manejo de la información debe darse desde los niveles básicos de la enseñanza hasta los niveles superiores de nuestras universidades.

Así pues, la formación de usuarios implementada desde los niveles básicos del sistema educativo y continuada en los subsecuentes, contribuirá a optimizar el uso de la información, produciendo incrementos significativos en la investigación en todos los campos del conocimiento humano y propiciará el desarrollo de la sociedad.

Reconociendo la importancia del Programa General del Información de la Unesco, la formación del usuario incluye además "todo proyecto o programa destinado a orientar e instruir a los usuarios, individual o colectivamente, con el objetivo de facilitar el reconocimiento de sus propias necesidades de información, la formulación de estas necesidades, la utilización efectiva y eficaz de los servicios de información así como la evaluación de estos servicios". 16

Por medio de la formación de usuarios de la información, el estudiante estará motivado para adentrarse en el proceso de búsqueda y recuperación de los recursos y medios que requiera para su labor académica y desarrollo personal y/o profesional.

Esto le hará desarrollar también actitudes positivas con relación a la biblioteca y demás unidades de información, así como de utilizar tal recurso.

La formación de usuarios de la información debe plantearse como un conocimiento indispensable que debe adquirirse en los niveles básicos (primaria y secundaria) y medio superior (preparatoria), que facilitará el de-

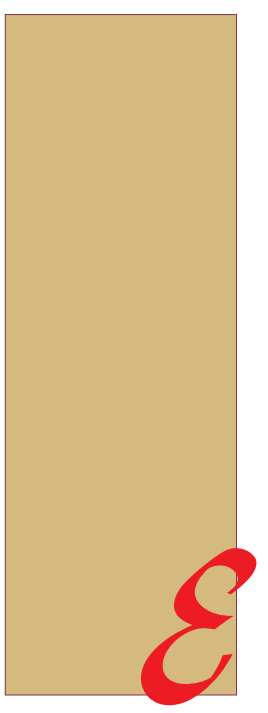

sarrollo del estudiante en el nivel superior (licenciatura) y lo preparará, en última instancia, para un mejor desempeño de su ejercicio profesional.

Ahora bien, en los países desarrollados, como los Estados Unidos, existe conciencia de parte de los planificadores de los sistemas educativos, sobre la importancia de la formación de usuarios, y la incluye dentro de los planes y programas de estudio, desde los niveles escolares elementales poniendo énfasis en la formación práctica en los niveles superiores. Tal es el caso de las universidades de Míchigan, Rochester, Columbia, Maryland y Harvard, en las cuales se encuentran antecedentes en este sentido desde $1880 .{ }^{17}$

El sistema educativo norteamericano considera que la formación del usuario es parte inseparable del programa general de instrucción, por lo que el curso de instrucción en el manejo de la biblioteca y sus fuentes de información, debe ser planeado de manera científica e implementarse en forma sistemática. ${ }^{18}$

Lamentablemente los países en desarrollo (particularmente el nuestro) no han considerado la formación del usuario como parte integrante del programa general del sistema educativo de manera sistemática. Existen en cambio tanto en el nivel medio como superior, asignaturas tales como: español, taller de investigación, técnicas de investigación documental, sistemas de información, metodología de las ciencias sociales, taller de redacción entre otras, que de alguna manera contemplan actividades relacionadas con el uso de la biblioteca, el manejo de bibliografías, recuperación de la información, elaboración y uso de diferentes tipos de fichas, etcétera, pero totalmente aisladas, que en el fondo son actividades que no tienen como objetivo primordial lograr la formación de usuarios de la información sino más bien, cumplir con los objetivos específicos de la asignatura en cuestión; es decir, no constituyen un contenido formal, independientemente de otras que están dirigidas a la formación de los usuarios, en la localización, manejo y uso de la información. 
La ausencia de una tradición bibliotecaria y la carencia de programas y políticas educativas, a nivel nacional, tendientes a la promoción y establecimiento de bibliotecas escolares, así como la carencia de un hábito de lectura en la población mexicana, no han permitido la comprensión y el gradual desarrollo de lo que implica la formación del usuario, las ventajas del buen uso y manejo de la información desde los niveles básicos de educación.

Esta falta de tradición en el país ha dejado como experiencia que un buen número de nuestros estudiantes universitarios carezcan de una conciencia y no den a la biblioteca y demás unidades de información el valor real de sus recursos y posibilidades de desarrollo.

Tomando como parámetro el nivel educativo profesional (licenciatura) del sistema educativo más grande y complejo de nuestro país, como lo es la Universidad Nacional Autónoma de México, la cual sirve como modelo al resto de las universidades públicas estatales, se encontró que de un total de 60 carreras profesionales, solamente 4 de ellas han contemplado dentro de su estructura curricular, una asignatura con valor en créditos que se dedique con mayor profundidad a la formación del usuario en el área del conocimiento que se trate.19

En Ciudad Universitaria, la Facultad de Química ofrece de manera obligatoria la asignatura Información química bibliográfica en el sexto semestre, con valor de 6 créditos. La Facultad de Medicina Veterinaria y Zootecnia, de manera optativa, la asignatura Métodos y técnicas de la consulta bibliográfica, con valor de 2 créditos. En la Escuela Nacional de Estudios Profesionales, Unidad Acatlán, se identificaron 2 carreras: Ciencias Políticas y Administración Pública y Relaciones Internacionales; ambas con la asignatura optativa Sistemas de Información con valor de 8 créditos.
Por su parte el Instituto Politécnico Nacional, con un catálogo de 40 carreras profesionales, no ha incluido en el mapa curricular de éstas ninguna asignatura específica que aborde la temática de la formación del usuario de la información. Sólo se localizaron asignaturas relacionadas con la investigación documental en 8 carreras. 20

Otro sistema importante, reflejado por su cobertura en el Distrito Federal, así como por la matrícula y la calidad académica de sus programas lo representa la Universidad Autónoma Metropolitana. Esta Universidad proporciona un total de 55 carreras a nivel profesional, de las cuales sólo 13 contemplan asignaturas relacionadas con la investigación documental, sin que ellas tengan relación directa con la formación del usuario. 21

Como puede observarse, de este gran total de 155 carreras, impartidas en tres de las más grandes instituciones de educación superior del país, un escaso número de ellas contemplan asignaturas que formen al usuario de la información. El caso más singular lo representa la Facultad de Química de la UNAM, la cual cuenta con un programa específico dirigido a la especialidad, al uso y manejo de fuentes de consulta en el área, así como a una carga teórico-práctica que le permite al estudiante un refuerzo evidente en su desarrollo profesional. Considerando lo anterior, así como las actividades tendientes a la formación de usuarios que cada una de las bibliotecas e instituciones han promovido en este sentido, es importante señalar que programas como los establecidos en la Facultad de Química o los de la ENEP-Acatlán, o bien el programa optativo de la Facultad de Medicina Veterinaria y Zootecnia de la UNAM, son esfuerzos importantes que muestran la necesidad de programas formalmente establecidos.

Implementar la formación de usuarios de la información dentro del proceso enseñanza-aprendizaje, en todos los niveles del sistema educativo, es un hecho que ha reconocido el Programa
General de Información de la Unesco, al señalar que "...Así como en la introducción de una enseñanza sistemática sobre la utilización de los recursos de información en los planes de estudio de las universidades, escuelas técnicas y otras instituciones educativas", ${ }^{22}$ es de primordial importancia atacar de raíz el problema de la formación de usuarios de la información, proporcionándola a través de la inclusión en la currícula profesional, de asignaturas específicas que la contemplen. Para tal caso, se propone un bosquejo que bajo el nombre genérico de "Recomendaciones para un programa universitario de formación de usuarios" agrupa una amplia tarea que comprende el desarrollo de actividades del usuario con relación a la biblioteca y a la búsqueda de información; así como el entrenamiento en el uso de la propia biblioteca, de sus recursos de información y la instrucción bibliográfica.

De esta forma estaríamos en concordancia con lo estipulado por Ricardo Gietz, ${ }^{23}$ cuando señala que en la formación al usuario hay que:

1.Sensibilizarlo para que pueda conocer sus necesidades de información.

2.Motivarlo para que adopte una posición activa frente al problema.

3.Dotarlo de de los elementos básicos para el conocimiento de los caminos y las herramientas para que se facilite el acceso a la información.

Al considerar estos aspectos también se está cumpliendo con lo expuesto por Beverly Lynch,24 cuando identifica cuatro principios básicos para el éxito de los programas de formación de usuarios:

1. Debe ser considerado como un proceso esencial dentro de su formación académica.

2. Debe ser diseñado dentro del contexto de un curso en particular o en un programa académico y debes ser con- 
sistente con el programa educativo dentro del cual está insertado.

3. Debe ser estructurado en función de las necesidades de los usuarios y los requerimientos de uso de determinadas herramientas de información.

4. La enseñanza de habilidades para el uso de la biblioteca debe mostrar un progreso en los estudiantes, es decir, ir de lo más sencillo a lo más complejo.

Es de esta manera, que el programa que se propone desarrollar, responde a necesidades concretas, lo cual variará de acuerdo a las categorías de usuarios y a los recursos económicos, tecnológicos y humanos disponibles.

\section{RECOMENDACIONES PARA UN PROGRAMA UNIVERSITARIO DE FORMACIÓN DE USUARIOS}

USUARIOS: Este programa está destinado a los alumnos que inician su nivel superior, los cuales se caracterizan porque inician el estudio de materias directamente relacionadas con el desarrollo de su profesión. Constituyen una gran población.

CONTENIDO: Deberá promover el conocimiento a fondo de la biblioteca y demás unidades de información, de las fuentes de información y de los instrumentos para recuperarla. La característica del contenido va a estar determinada por la especialización de área de que se trate (biología, filosofía, derecho, economía, arquitectura, matemáticas, etcétera).

Parte de este contenido deberá darse con la temática:

1. Concepto, importancia y generación de la información.

2. Información y recuperación en documentos primarios.

3. Información y recuperación en documentos secundarios.

4. Transferencia y distribución de la información bibliográfica (del área que se trate).

5. Organización y sistematización de la información bibliografía (del área que se trate).

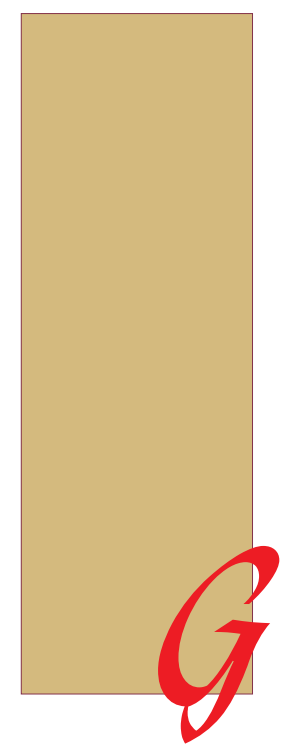

Con el desarrollo de un programa de formación de usuarios de la información a nivel universitario se cubriría gran parte de las deficiencias que en este rubro se han presentado.

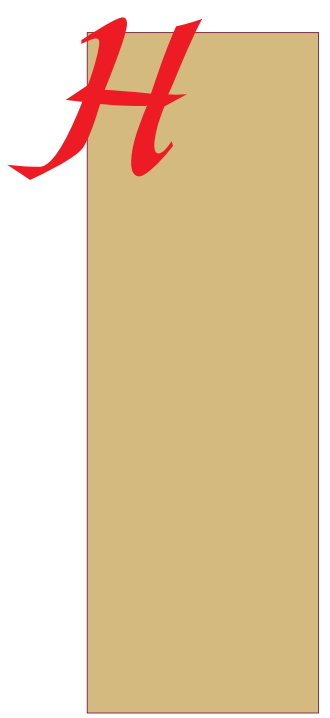

6. Aprovechamiento y recuperación de la información bibliográfica, ya sea por medios manuales o automatizados (del área que se trate).

7. Manejo y uso de las diferentes tecnologías de la información (del área que se trate).

OBJETIVOS: Que el estudiante adquiera las habilidades y destrezas suficientes para:

1. Utilizar exhaustivamente las bibliotecas y demás sistemas de información, nacionales e internacionales.

2. Satisfacer la búsqueda exhaustiva bibliográfica y de documentos para la realización de sus trabajos escolares, tesis e investigación.

3. Conocer y manejar las fuentes de información especializada, tales como: bibliografías, índices, resúmenes, etcétera (del área que se trate).

IMPARTICIÓN: Los contenidos de este programa deberán incluirse y desarrollarse preferentemente dentro de una asignatura de nueva creación, cuyo nombre podría ser: "Información bibliográfica y documental", con el agregado de la especialidad de que se trate. Ej. Información bibliográfica y documental física...Información bibliográfica y documental económica...etcétera. Dicha asignatura deberá ser de carácter obligatorio y con valor en créditos e impartirse desde el segundo semestre de la carrera. Tendrá una duración mínima de dos semestres.

La impartición de tal asignatura estará a cargo y responsabilidad de un profesor especializado en el área en cuestión, con conocimientos sólidos del contenido señalado, o bien de un bibliotecario profesional con la especialidad del área.

AUXILIARES: Se podrá hacer uso de los medios impresos, visuales y audiovisuales que determine el profesor responsable de la materia. Para esta asignatura serán indispensables las obras de consulta (primarias o secundarias, impresas o electrónicas, etcétera) de la especialidad de que se trate. 


\section{TÉCNICAS DE ENSEÑANZA:}

Se empleará la clase dentro del aula apoyándose en la biblioteca como laboratorio de prácticas.

De igual forma, serán importantes las asesorías, sesiones prácticas visitas guiadas, demostraciones, seminarios, conferencias, etcétera, así como algunos manuales de autoinstrucción.

\section{IMPLEMENTACIÓN:}

Para estos efectos, cada Escuela o Facultad, o en su defecto cada Licenciatura (en caso de ser necesario), deberá proponer la modificación a su plan de estudios, con la finalidad de incluir en él la asignatura que desarrolle los contenidos anotados y referidos a la formación de usuarios. Para ello deberán crearse equipos multidisciplinarios que se encarguen de la elaboración, estructura y alcances de la nueva asignatura.

Se recomienda que esta asignatura sea revisada y cuente con el aval de órganos académicos reconocidos de la especialidad, tales como las escuelas de bibliotecología, el Centro Universitario de Investigaciones de la UNAM.

\section{EVALUACIÓN:}

Deberá efectuarse a través de los métodos y mecanismos de evaluación empleados para cualquier otra asignatura de la Escuela o Facultad de que se trate.

Finalmente, considero que con el desarrollo de un programa de formación de usuarios de la información a nivel universitario, cubriremos gran parte de las deficiencias que en este rubro se han presentado.

Con este programa evitamos, en gran medida, el desperdicio y subutilización de los recursos de información, contribuirá al desarrollo nacional y logrará en buena parte, desarrollar en el usuario la autosuficiencia informativa al traspasar los complicados y complejos sistemas que utilizan las bibliotecas y demás unidades de información. “... Nada nos causará mayor satisfacción que ver a nuestros usuarios manejar las fuentes de información por sí mismos y que sólo recurran a nosotros en los casos verdaderamente difíciles; en otras palabras, tener usuarios autosuficientes en el uso de las bibliotecas y fuentes de información". 25

\section{NOTAS BIBLIOGRÁFICAS}

1. HONORE, Bernard. Para una teoría de la formación: dinámica de la formalidad. Madrid: Nancea, 1980. 170 p.

2. LHOTELLIER, A. "La formation en question". En Formation. No, 1. 1974. pp. 47-72.

3. COOPER, D. La muerte de la familia. Barcelona: Ariel, 1978. 112 pp.

4. MENZE, Clemens. "Formación". En: Conceptos fundamentales de pedagogía. Barcelona: Herder, 1981. pp. 267-297.

5. HONORE. Op. Cit.

6. MENZE. Op. Cit.

7. VID. CONFERE. Conceptos fundamentales de pedagogía. Compilado por Josef Speck y otros. Barcelona: Herder, 1981. 347 p.

8. VID. LONNGI, Natalia. "Algunas consideraciones sobre el usuario y el servicio de consulta." En: Encuentro de Bibliotecarios de la Universidad Nacional Autónoma de México ( $1^{\circ} .:$ 1982: México). Prospectiva de la biblioteca universitaria. México: UNAM, DGB, 1983, pp. 453-466.

SMITH ABRUCH, Sonia. "Algunas consideraciones sobre el usuario y el servicio de consulta". En: Encuentro de Bibliotecarios de la Universidad Nacional Autónoma de México. (1º: 1982: México). Prospectiva de la biblioteca universitaria. México: UNAM, DGB, 1983, pp. 277-286. 
9. Encuesta piloto realizada durante septiembre-noviembre de 1991 en Escuelas y Facultades de la UNAM, Los resultados fueron presentados en el seminarios de Investigación de Educación de Usuarios del Centro Universitario de Investigaciones Bibliotecológicas de la UNAM, el día 31 de enero de 1992.

10. Encuesta aplicada de noviembre de 1991 a enero de 1992 directamente a bibliotecas de distintas universidades del país que cuentan con alguna actividad tendiente a la formación usuarios: Universidad de Colima, Universidad Autónoma de Baja California Sur, Universidad Autónoma de Nuevo León y Escuelas y Facultades de la UNAM.

Los resultados fueron presentados en el Seminario de Investigación de Educación de Usuarios del Centro Universitario de Investigaciones Bibliotecológicas de la UNAM, el día 7 de febrero de 1991.

11y 12. Se refiere a la encuesta piloto y de aplicación de cuestionarios citados (10 y 11), que incluyó un cuestionario para usuarios.

13. LONNGI y ABRUCH. Op. Cit. VELEZ, Cecilia. "Programa de educación de usuarios de la Facultad de Psicología de la UNAM" En Encuentro de Bibliotecarios de la Universidad Nacional Autónoma de México. (2 ${ }^{\circ}$ : 1983: México). Significado y alcance de la biblioteca universitaria en la sociedad mexicana: memoria. México: UNAM, DGB, 1984. pp. 349-354.

14. UNAM. Dirección General de Bibliotecas. Ven y utiliza los recursos de tu biblioteca. México: La Dirección, 1992. 39 p. (Folleto complementario a los videos: Ven a tu biblioteca y utiliza los recursos de tu biblioteca. Preparados por la propia DGB-UNAM.

15. SOSA ESQUIVEL, Noemí. "El entrenamiento de usuarios en instituciones de investigación: el caso del IIE". En: Fornadas Mexicanas de Biblioteconomía (17 as.: 1986: Puebla, Pue.) México: SEP, DGB, 1988. pp. 131-141.

16. TOCATLIAN, Jacques. "Formación de usuarios de la información: programas, problemas y perspectivas" En: Boletín de la Unesco para las bibliotecas. V. 32. No. 6. 1978. pp. 382-390.

17. User instruction in academia libraries: a century of selected readings. Compiled by Larry L. Hardesty et al. Matuchen, N.J. and London: Scarecrow Press, 1986. 311 p.

18. DAVIS, Ruth Ann. "Educating library users in the senior high school" En. Lubans, John Jr. Educating the library users. New York: Bowker, 1974. pp. 40-45.

19. UNIVERSIDAD NACIONAL AUTÓNOMA DE MÉXICO. Catálogo general. 1989. México; La Universidad, 1989.

20. INSTITUTO POLITÉCNICO NACIONAL. Guía de carreras profesionales. 1984-1985. México: El Instituto, 1986. 
21. UNIVERSIDAD AUTÓNOMA METROPOLITANA. Catálogo general. 1989. México; La Universidad, 1989.

22. TOCATLIAN. Op. Cit.

23. GIETZ, Ricardo A. La formación y el adiestramiento del investigador como usuario de la Información. En: Seminario Latinoamericano sobre Preparación de Científicos de la Información. México: CONACYT, 1972.

24. LYNCH, Beverly P. The involvement of the librarians in the total education process. En: Library Trends. V.29 No. 1, 1980.

25. CASTILLO SÁNCHEZ, Octavio, "Educación/entrenamiento de usuarios: por quién y para qué”. En: Revista ALEBCI v. 1, No. 1, 1987. pp. 81-121. 